ORIGINAL RESEARCH

\title{
Microbiome Profile in Chronic Rhinosinusitis with and without Polyps of Makassar, Indonesia
}

Azmi Mir’ah Zakiah¹, Muhammad Fadjar Perkasa², Amelia Dian Utami ${ }^{3}$, Riskiana Djamin ${ }^{4}$, Burhanuddin Bahar ${ }^{5}$, Firdaus Hamid ${ }^{6}$, Abdul Qadar Punagi ${ }^{7}$

\begin{abstract}
Background: Chronic rhinosinusitis (CRS) is a prevalent health problem that results in a large costly burden in society is often associated with the role of the microbiome that seems to maintain a healthy state and stability of the sinonasal environment often viewed as a symbiotic system. Aim and objective: This study aimed to identify a microbiome profile in chronic rhinosinusitis with and without nasal polyps of Indonesian population.
\end{abstract}

Materials and methods: This study was conducted using the case-control technique on 20 patients divided into two groups. The first group is CRS without nasal polyps consisted of 10 patients and the second group is CRS with nasal polyps consisted of 10 patients. All of the samples were examined by next-generation sequencing techniques.

Results: Microbes were detected in all samples. Actinobacteria, Bacteroidetes, Cyanobacteria, Firmicutes, Fusobacteria, and Proteobacteria were the dominant phylum in both groups with a variable number of percentages.

Conclusion: This study highlights alteration of the commensal microbe may lead to dysbiosis conditions of the sinonasal environment.

Keywords: Chronic rhinosinusitis, Microbiome, Nasal polyps.

Otorhinolaryngology Clinics: An International Journal (2019): 10.5005/jp-journals-10003-1337

\section{INTRODUCTION}

Chronic rhinosinusitis (CRS) is an inflammation of the nose and paranasal sinuses lasting for $>3$ months without resolution of symptoms. ${ }^{1}$ According to the European Position Paper on Rhinosinusitis and Nasal Polyps (EPOS) in 2012, adult rhinosinusitis characterized by two or more symptoms, which should be either nasal obstruction or nasal discharge with/without facial pain or reduction of smell and also an endoscopic sign of nasal polyps, mucopurulent discharge from middle meatus or mucosal obstruction in middle meatus and computed tomography (CT) changes. Chronic rhinosinusitis is classified as two clinical phenotypes, CRS with nasal polyps (CRSwNP) and CRS without nasal polyps (CRSsNP). ${ }^{2}$

Chronic rhinosinusitis is a prevalent health problem that results in a large costly burden in society. Current data have demonstrated that CRS was found in approximately $5-15 \%$ of the general population. ${ }^{3}$ It affects $14-30$ million US adults per year, which results in 13 million office visits per year and spent $\$ 3.4-8.6$ billion annually. ${ }^{4}$

Over a decade, several hypotheses have been proposed to explain CRS pathogenesis. Recent studies revealed that the sinonasal cavity has a resident flora, similar to the gut, which helps to promote a sinonasal healthy environment. Numerous studies were conducted to understand this phenomenon to attempt and define human microbiome in the sinonasal cavity. ${ }^{5}$ Microbiome refers to the sequence of genes encoded by microorganisms that inhabit the environment. ${ }^{6} \mathrm{~A}$ new understanding is arising in the etiology and pathogenesis through a concept of important interaction of the human body with the microbiome. ${ }^{7}$

The commensal microbial community seems to maintain a healthy state and stability of the sinonasal environment often viewed as a symbiotic system. A diverse commensal microbiome
${ }^{1-4,7}$ Department of Otolaryngology-Head and Neck Surgery, Medical Faculty, Hasanuddin University, Makassar, Indonesia

${ }^{5}$ Department of Nutrition, Public Health Faculty, Hasanuddin University, Makassar, Indonesia

${ }^{6}$ Department of Microbiology, Medical Faculty, Hasanuddin University, Makassar, Indonesia

Corresponding Author: Azmi Mir'ah Zakiah, Department of Otolaryngology-Head and Neck Surgery, Medical Faculty, Hasanuddin University, Makassar, Indonesia, Phone: +6287840717545, e-mail: mimz.aspar712@gmail.com

How to cite this article: Zakiah AM, Perkasa MF, Utami AD, et al. Microbiome Profile in Chronic Rhinosinusitis with and without Polyps of Makassar, Indonesia. Int J Otorhinolaryngol Clin 2019;11(3):55-63.

Source of support: Nil

Conflict of interest: None

consortium contributes many useful functions for the sinus. The healthy condition of the microbiome has an important interaction with pattern recognition receptor (PRR) on epithelial which adjusts the relationship between pro- and anti-inflammatory cytokines, chemokines, and mediators. Changes in the sinonasal microbiome resulting in "dysbiosis" condition. A shift from a normal healthy microbial community may responsible for modulating sinuses inflammation through any number of mechanisms. Pathogen colonization and disproportion of microbiota leading to the dysfunctional immune barrier, inflamed mucosal epithelium, and obstructed sinuses promote a suitable condition for dysbiosis, setting the stage for CRS. ${ }^{7-10}$

Microbiome identification using culture-independent techniques has emerged due to the understanding of the role of microbes in human well-being. ${ }^{11}$ Culture-independent methods allow identification of microorganisms without requiring growth 
in vitro and even if they are non-viable and based on the direct analysis of bacterial DNA (or RNA). ${ }^{6,10,11}$

The newest method to specify the composition of the microbiome is next-generation sequencing, which is designed to identify a cluster of 16 s ribosomal RNA (rRNA) that are specific to a typical genus or species called operational taxonomic units. ${ }^{4}$

The primary aim of this study is to profile the microbiome in CRS patients with and without nasal polyps in Indonesia.

\section{Materials and Methods}

\section{Ethics Statement}

The study protocol and ethical clearance were approved by the Biomedical Research Ethics Committee on Human Faculty of Medicine Hasanuddin University, Makassar, Indonesia (Register number: 14/UN4.6.4.5.31/PP36-KOMETIK/2019 and protocol number: UH18120957). All participants provided written informed consent before they participated in the project.

\section{Study Design and Population}

Study participants were recruited in 2019 at Wahidin Sudirohusodo Hospital, Hasanuddin University Hospital, and Mitra Husada Hospital Department of Otorhinolaryngology, Head and Neck Surgery, Hasanuddin University, Makassar, Indonesia. Two groups of participants (CRSsNP and CRSwNP) in equal number were informed about the study and invited to participate: Both the groups scheduled for endoscopic sinus surgery on all paranasal sinuses for the treatment of CRS. Diagnosis of CRS was made according to the European Position Paper on Rhinosinusitis and Nasal Polyps 2012. ${ }^{2}$

\section{Sample Collection}

Intraoperatively, through modern techniques of Functional Endoscopic Sinus Surgery (FESS), the mucosa was preserved while creating the largest possibly sinus cavities enlargement of natural ostia, the uncinate process was excised with cutting instruments. Care was taken to avoid contamination from other mucosal sites. Each nasal tissue sample was placed in a sterile Eppendorf tube contains $1 \mathrm{~mL} \mathrm{DNA} / \mathrm{RNA}$ shield ${ }^{\mathrm{TM}}$ solution and stored in $-20^{\circ} \mathrm{C}$ until further analysis in Novogene AIT Laboratory, Singapore with several reports H101SC19020094.

\section{DNA Extraction}

Total genome DNA from samples was extracted using the CTAB/ SDS method. DNA concentration and purify was monitored on $1 \%$ agarose gels. According to the concentration, DNA was diluted to $1 \mathrm{ng} / \mu \mathrm{L}$ using sterile water.

\section{Library Preparation and Sequencing}

Amplification of the $16 \mathrm{~S}$ rRNA gene $\mathrm{V} 3-\mathrm{V} 4$ region was performed by using specific primers designed to anneal to $341 \mathrm{~F}$ (5'-CCTACGGGNGGCWGCAG-3') and $806 \mathrm{R}$ (5'-GGACTACNNGGGTATCTAAT-3'). All PCR reactions were carried out with Phusion ${ }^{\circledR}$ High-Fidelity PCR Master Mix (New England Biolabs). This resulted in amplicon sizes of approximately $466 \mathrm{bp}$. All further steps in library preparation were performed according to the Illumina "16S Metagenomic Sequencing Library Preparation" protocol.

Shortly, PCR Clean-up, Index PCR, PCR Clean-up 2, library quantification, normalization, and pooling were performed according to the above-referred manual. The libraries were generated with lon Plus Fragment Library Kit 48 rxns for Thermo fisher and quantified via Qubit and Q-PCR. Would be sequenced by IonS5 ${ }^{\mathrm{TM}} \mathrm{XL}$ (Thermo fisher).
Single-end reads were assigned to samples based on their unique barcode and truncated by cutting off the barcode and primer sequence. Quality filtering on the raw reads was performed under specific filtering conditions to obtain the high-quality clean reads according to the Qiime (v.1.7.0) quality-controlled process. The reads were compared with the reference database using the UCHIME algorithm to detect chimera sequences. And then the chimera sequences were removed, the effective reads finally obtained.

\section{Sequence Analysis}

Sequences analysis was performed by Uparse software (Uparse v.7.0.1001) using all effective reads. Sequences with $\geq 97 \%$ similarity were assigned to the same OTUs. The representative sequence for each OTU was screened for further annotation. For each representative sequence, Morthur software was performed against the SSUrRNA database of SILVA Database for species annotation at each taxonomic rank. To get the phylogenetic relationship of all OTU representative sequences, the MUSCLE can compare multiple sequences rapidly. OTU abundance information was normalized using a standard of sequence number corresponding to the sample with the least sequences. Subsequent analysis of alpha diversity and beta diversity were all performed basing on this output normalized data.

Alpha diversity (within samples) is applied to analyze the complexity of species diversity for a sample through three indices, including Observed_species, Chao1, and Shannon. All these indices were calculated with QIIME (v.1.7.0) and displayed with R software (v.2.15.3). Chao1 assesses the richness of species, Observed_species assess the amount of OTUs found in each sample, and the Shannon index assesses the diversity of species. ${ }^{12}$

Beta diversity analysis (among samples) on both weighted and unweighted UniFrac was calculated by QIIME software (v.1.7.0). Principal Coordinate Analysis (PCoA) analysis was displayed by the WGCNA package, stat packages, and gg plot2 package in R software (v.2.15.3). Unweighted Pair-Group Method with Arithmetic Means (UPGMA) was conducted by QIIME software (v.1.7.0).

$p$ value was calculated by the method of the permutation test while the $q$ value was calculated by the method of Benjamin and Hochberg false discovery rate. ANOSIM and MRPP were performed by R software. ANOSIM analysis is a non-parametric test to evaluate whether variation among groups is significantly larger than variation within groups, which helps to evaluate the reasonability of the division of groups. MRPP is a non-parametric procedure to test the hypothesis of no difference between two or more groups of entities and can analyze comparisons between all groups. The MRPP is similar to ANOSIM, which aims at determining whether the difference of microbial community structure among groups is significant. The MRPP calculates the average intragroup distance between samples and compares it with the average intergroup distances, providing a measure of dissimilarity using a "delta score".

ANOSIM and MRPP analysis could be used to determine whether community structure significantly differs between groups, or comparing the differences between groups and within groups.

\section{Results \\ Patients Materials}

The study participants comprise 20 subjects, including 10 CRSsNP patients (NP group samples), and 10 CRSwNP (P group samples) patients. All patients undergoing FESS. Fifty percent $(n=10)$ of CRS 
Table 1: Distribution of gender, age, and phenotype of the study participants

\begin{tabular}{lll}
\hline Variable & $n(20)$ & $\%$ \\
\hline Age range & & \\
18-28 years & 10 & 50 \\
29-39 years & 6 & 30 \\
40-50 years & 1 & 5 \\
$>50$ years & 3 & 15 \\
Gender & & \\
Male & 9 & 45 \\
Female & 11 & 55 \\
Phenotype & & \\
CRSsNP & 10 & 50 \\
CRSwNP & 10 & 50 \\
\hline
\end{tabular}

Table 2: Summary of the effective tags and OTUs

\begin{tabular}{llllll}
\hline Sample_Name & Total_tags & Taxon_Tags & Unclassified_Tags & Unique_Tags & OTUs \\
\hline P1 & 90,253 & 54,445 & 8,182 & 27,626 & 2,008 \\
P2 & 68,327 & 51,731 & 3,251 & 13,345 & 1,278 \\
P3 & 90,104 & 59,524 & 1,667 & 28,913 & 1,475 \\
P4 & 57,516 & 5,892 & 26,751 & 1,999 \\
P5 & 90,159 & 57,811 & 6,546 & 25,730 & 1,921 \\
P6 & 90,087 & 56,139 & 3,464 & 30,446 & 1,899 \\
P7 & 90,049 & 59,532 & 4,966 & 25,620 & 1,889 \\
P8 & 90,118 & 58,467 & 3,981 & 27,752 & 1,204 \\
P9 & 18,008 & 11,932 & 24,577 & 805 \\
P10 & 90,200 & 58,876 & 6,989 & 24,462 & 1,836 \\
NP1 & 54,517 & 64,807 & 1,662 & 23,726 & 1,156 \\
NP2 & 90,327 & 295 & 10,149 & 771 \\
NP3 & 90,195 & 3,602 & 3,799 & 3,241 & 2,183 \\
NP4 & 90,046 & 57,339 & 188 & 1,753 & 1,222 \\
NP5 & 91,379 & 61,708 & 5,794 & 22,660 & 1,381 \\
NP6 & 97,649 & 61,692 & 9,123 & 33,396 & 1,518 \\
NP7 & 90,146 & 47,051 & 6,519 & 22,424 & 1,059 \\
NP8 & 89,570 & 52,471 & 1,837 & 31,938 & 791 \\
NP9 & 81,414 & 58,137 & 379 & 31,990 & 2,079 \\
NP10 & 90,106 & 65,134 & 67,619 & 22,199 & 1,252 \\
\hline
\end{tabular}
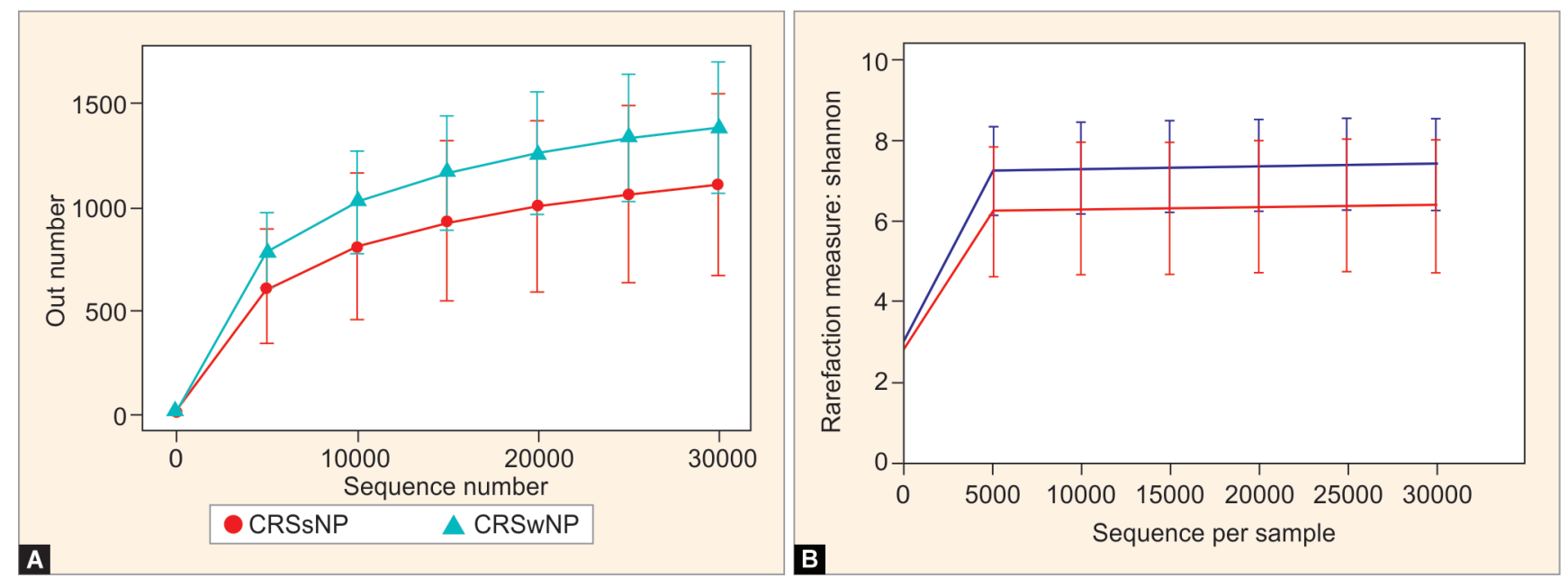

Figs $1 \mathrm{~A}$ and B: Rarefaction curves (A) and Shannon diversity curves (B) of DNA sequences of the sample. Curves were calculated based on OTUs at $97 \%$ similarity 
The rarefaction curves did not approach a plateau but Shannon's diversity rarefaction curves approached asymptotes (Fig. 1), indicating that the sampling depths were sufficient to capture the overall microbial diversities in all two groups. Shannon

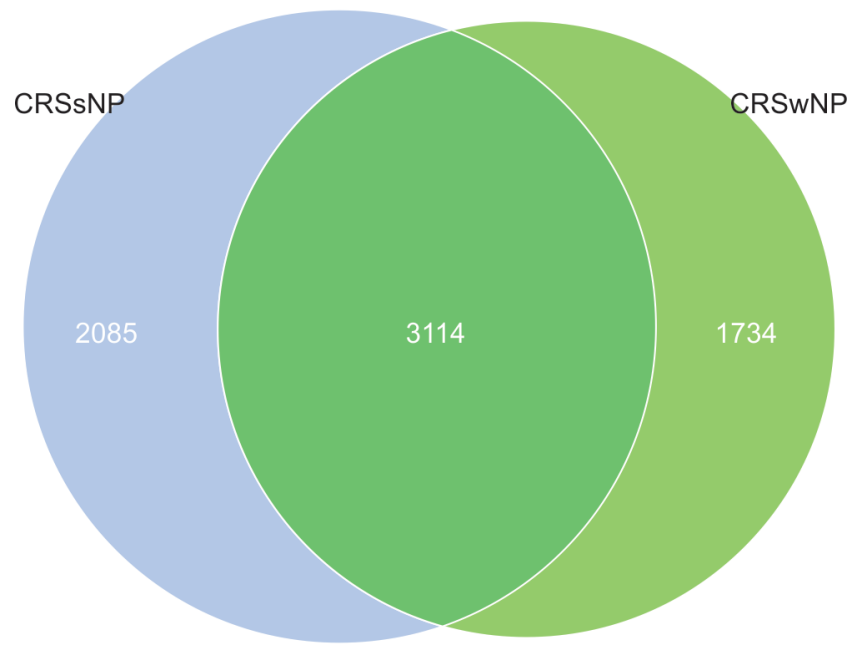

Fig. 2: Venn diagram of the OTUs in two groups. The number inside the diagram indicates the number of OTUs

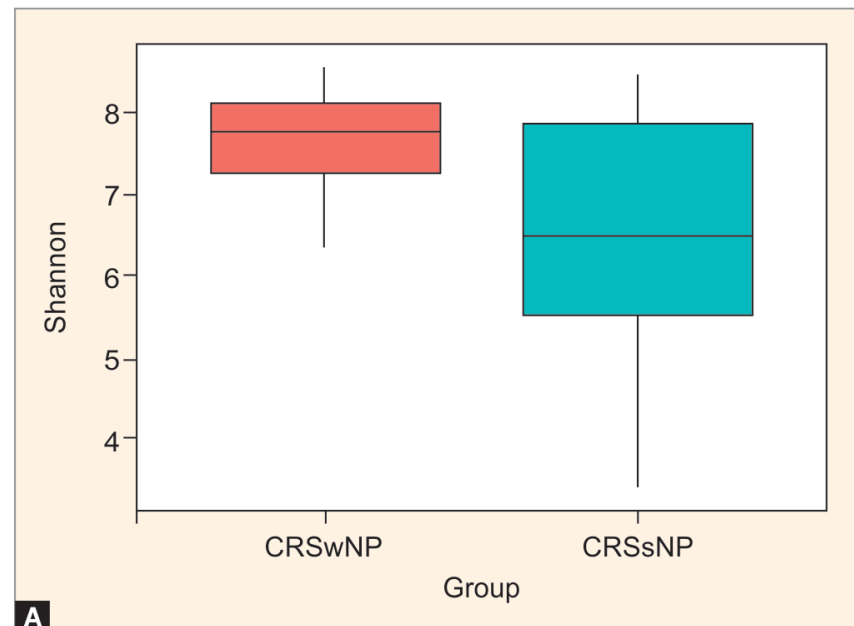

A

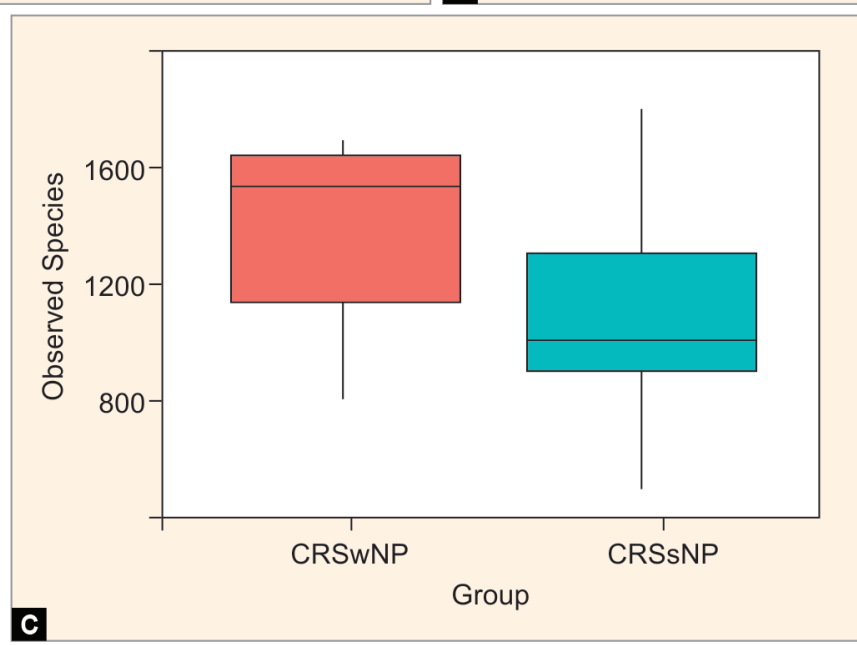

Figs 3A to C: (A) Boxplot of Shannon diversity indices; (B) Chao1 indices; (C) Observed species indices diversity indicates the diversity from the higher was sample NP $6(8,459)$ and the lowest was sample P8 $(6,386)$. Venn diagram displayed that CRSsNP and CRSwNP shared 3,114 OTUs (Fig. 2).

Differences in Shannon diversity were examined between the CRSsNP and CRSwNP groups. The result was CRSwNP had significantly higher diversity than CRSsNP, same result showed in Observed_species indices and Chao1 indices (Fig. 3).

Observed_species and Chao1 reveal the richness of species within a single sample, while Shannon indexes reflect microbial diversity as shown in Table 3. Observed_species, Chao1, and Shannon indexes in the CRSwNP group were higher than in the CRSsNP group, even though there was no statistical difference in alpha diversity between these two groups ( $p$ value $>0.05$ ).

We examined beta diversity in both groups, two dimensional Principal Coordinates Analysis (PCoA) of weighted and unweighted UniFrac distances were performed to conceive the relationship of the microbial communities in both groups of the sample (Fig. 4). Based on unweighted UniFrac PCoA (Fig. 4A), there are some bacterial communities of both groups were group together whereas some other bacterial communities were separated from each other. Based on the weighted UniFrac PCoA, both groups were spreading to the left and right groups. According to weighted UniFrac distance cluster analysis, which showed that 20 samples were clustered into a few groups. At the phylum level, 10 representatives phyla were identified, and the rest of the tags are indicated as "Others" (Fig. 5).

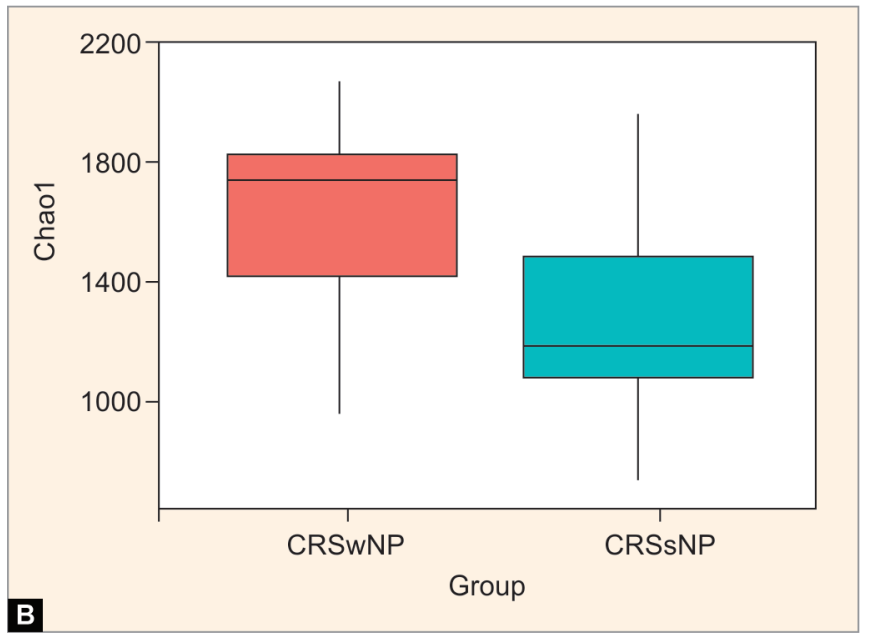


The mean weighted UniFrac and unweighted UniFrac in the CRSwNP group distances lower than the CRSsNP group but not statistically significant ( $p$ value $>0.05$; Fig. 6 ).

The ANOSIM analysis reveals a negative $R$ value $(-0.002)$ indicates that inner group variation is larger than intergroup variations, therefore no significant differences ( $p$ value $0.461>$ 0.05 ) between the CRSsNP and CRSwNP groups. Meanwhile, the MRPP analysis result shows the $A$ value $<0(-0.0024)$ represented that variation within the group is larger than among the group. The observed delta is smaller indicates that inner-group variation is small, while the larger one in the expected delta column means that

Table 3: Summary of the richness and diversity of microbial communities in 20 samples

\begin{tabular}{llll}
\hline Sample & $\begin{array}{l}\text { Observed_spe- } \\
\text { cies }\end{array}$ & Shannon & Chao1 \\
\hline P1 & 1,670 & 8.322 & 1845.783 \\
P2 & 1,084 & 4.555 & 1431.089 \\
P3 & 1,265 & 7.772 & 1407.523 \\
P4 & 1,689 & 7.85 & 1919.888 \\
P5 & 1,604 & 8.215 & 1796.014 \\
P6 & 1,639 & 7.638 & 2078.222 \\
P7 & 1,654 & 7.772 & 1797.644 \\
P8 & 958 & 6.386 & 1137.637 \\
P9 & 805 & 7.149 & 954.188 \\
P10 & 1,553 & 8.584 & 1707.841 \\
NP1 & 950 & 6.434 & 1095.805 \\
NP2 & 487 & 3.337 & 712.919 \\
NP3 & 1,926 & 7.882 & 2145.556 \\
NP4 & 977 & 6.54 & 1198.942 \\
NP5 & 1,106 & 5.556 & 1291.153 \\
NP6 & 1,370 & 8.459 & 1561.19 \\
NP7 & 895 & 5.462 & 1077.081 \\
NP8 & 631 & 4.213 & 840.189 \\
NP9 & 1,803 & 8.164 & 1979.367 \\
NP10 & 1,022 & 7.738 & 1175.679 \\
\hline & & &
\end{tabular}
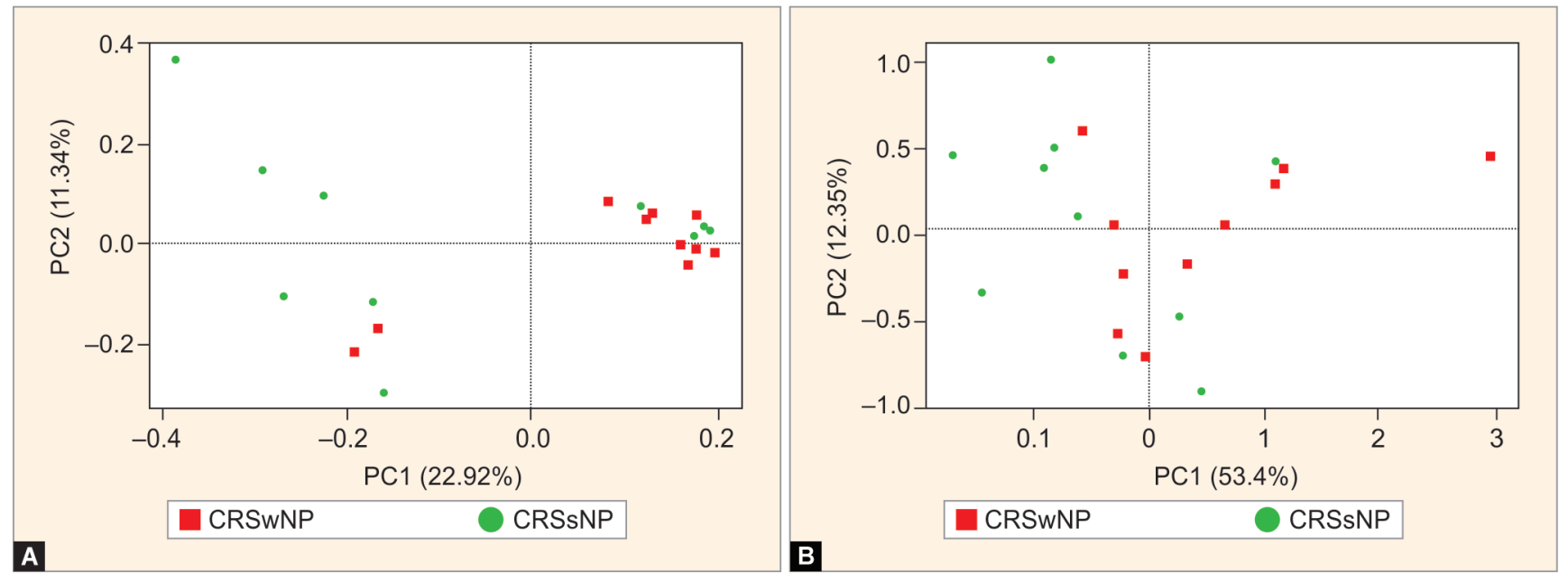

Figs 4A and B: Principal Coordinate Analysis (PCoA) of samples (A) based on unweighted UniFrac metrics of all samples. (B) PCoA plots are based on weighted UniFrac metrics of all samples intergroup variation is great. Therefore, no significant differences $(p$ value $0.597>0.05$ ) between CRSsNP and CRSwNP groups (Fig. 7).

\section{Microbial Identification}

At phylum level, there are six predominant phyla were identified in the CRSsNP group including Bacteroidetes (24.15\%), Proteobacteria (22.65\%), Cyanobacteria (18.83\%), Firmicutes (15.50\%), Fusobacteria (11.88\%), and Actinobacteria (6.99\%). While in the CRSwNP group, six predominant phyla including Proteobacteria (54.86\%), Firmicutes (21.06\%), Cyanobacteria (12.51\%), Fusobacteria (4.15\%), Actinobacteria (4.05\%), and Bacteroidetes (3.37\%) as shown in Table 4.

In the CRSwNP group, Proteobacteria phyla had the higher percentage and Bacteroidetes phyla had the less percentage, while in the CRSsNP group, Bacteroidetes phyla had the higher percentage and Actinobacteria phyla had the less percentage (Figs 8 and 9). There were no significant differences between both the groups of dominant phyla.

At the genus level, the 35 most abundant genera in the two groups were analyzed by a hierarchical clustering heat map, which denoted that although these genera occurred in all samples, the abundance of most genera varied in different samples. The heatmap of genera relative abundance was shown in Figure 10.

\section{Discussion}

This study investigated the bacterial microbiome of two groups of patients with CRS with and without nasal polyps by using $16 \mathrm{~s}$ ribosomal amplicon sequencing (next-generation sequencing) of the uncinate process of ostiomeatal complex.

There are two phenotypes of CRS that ruled in this study, most of the samples found in young and productive age (18-28 years), the prevalence was higher in females and we obtain an equal phenotype of CRS, 10 samples of CRSsNP and 10 samples of CRSwNP.

We speculated that CRS occurs at young and productive age because this group is more often exposed to environmental contaminants. While other studies showed the contradictive result, the incidence of CRS is between 37 years and 50 years old. Boase et al. found the incidence of CRS between 35 years and 47 years old Cleland et al. found the mean age of CRS incidence is 50 years old. ${ }^{14,15}$

Changes in alpha diversity represent the disease circumstances. In this study, we found Shannon diversity was examined between 


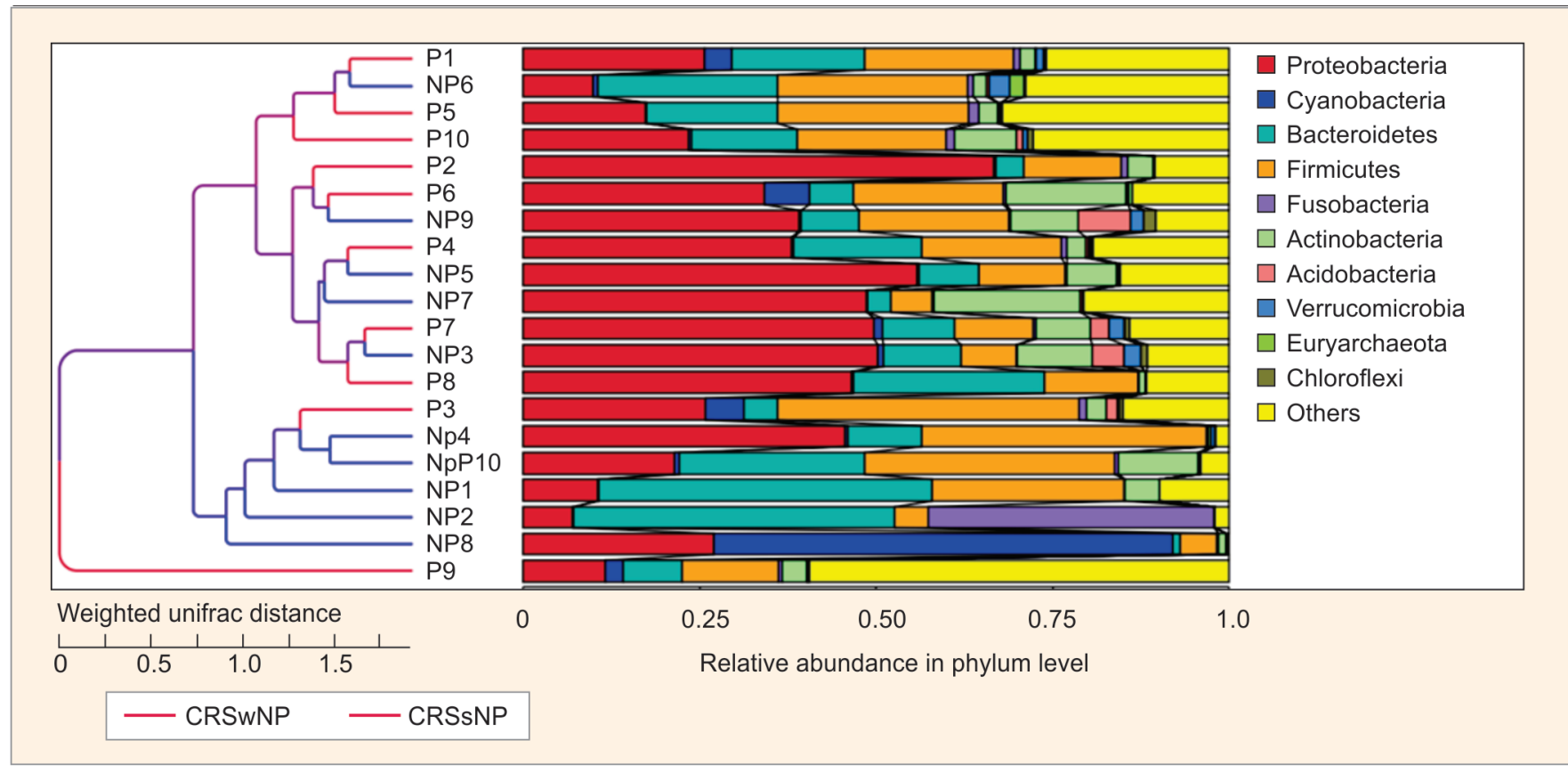

Fig. 5: Relative abundance of main bacterial communities in 20 samples at the phylum level. The horizontal coordinates are expressed the relative abundance and the longitudinal coordinate represents the samples. Each bar represents the average relative abundance of each bacterial taxon within a sample
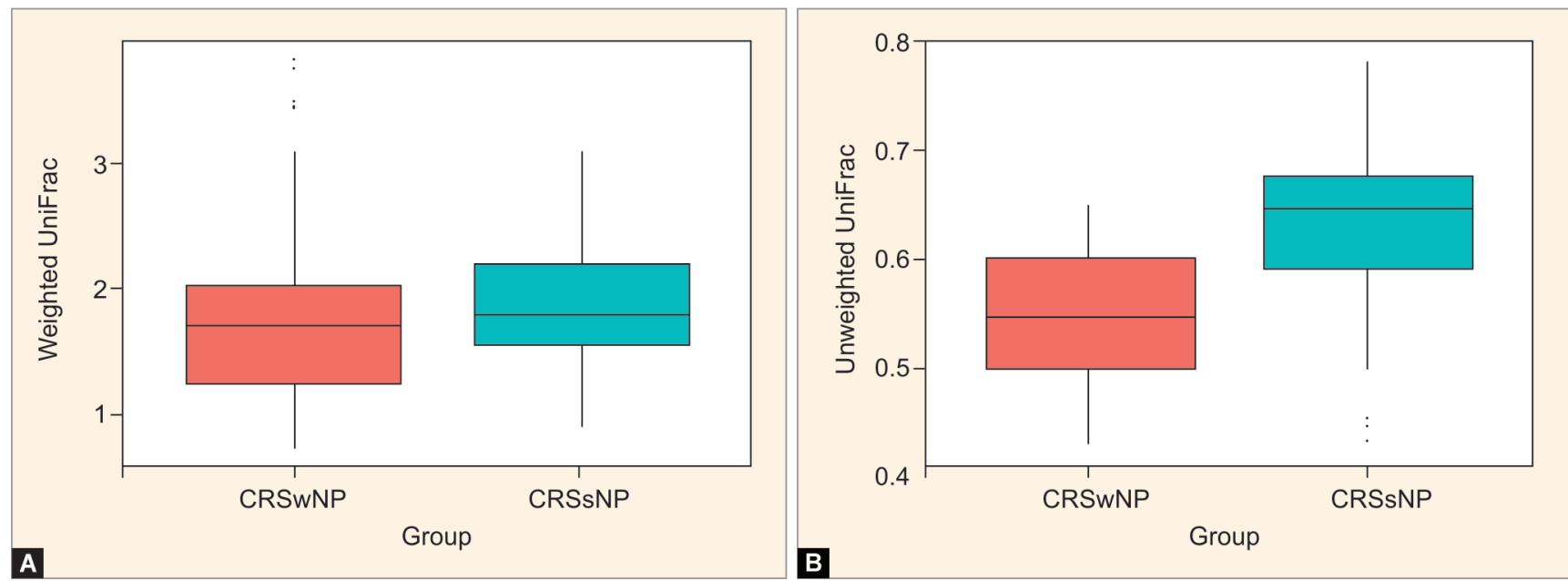

Figs 6A and B: Boxplots showing unweighted UniFrac and weighted UniFrac distance matrices. Both boxplots show no significant difference between both groups

the CRSsNP and CRSwNP groups and CRSwNP had significantly higher diversity than CRSsNP. The contrary result revealed by Copeland et al. showed the CRSwNP group had significantly lower diversity than CRSsNP. The presence of nasal polyps was associated with lower Shannon diversity when compared with CRSsNP. A previous study by Ramakrishnan et al. found that the existence of nasal polyps was not a forecaster of microbiome composition changes. ${ }^{9,10}$ We believed that the disparity results need more advanced study related to the presence or absence of allergy, asthma, eosinophilia or multiple inflammatory pathways in the CRSwNP group that can play role in altering the microbiome composition of this study. While in beta diversity used to compare microbial community structure within samples. In this study, innergroup variation is larger than intergroup variations. Hoggard et al. and Copeland et al. in their study explained that there was a tendency of higher intrapatient variability in the CRS group. We conceived larger sampling will explain this trend further. ${ }^{10,16}$

A previous study found that the phyla Firmicutes, Proteobacteria, and Actinobacteria were discovered in healthy subjects, whereas the phyla Firmicutes and Proteobacteria are less frequent. Bacteroidetes are also detected but in lower relative abundance. ${ }^{17,18}$ While Aurora et al. found that Cyanobacteria in large numbers. ${ }^{19}$ In our study, we did not compare healthy subjects with CRS, instead of CRSwNP and CRSsNP groups comparison. There were six predominant phyla in both CRSsNP and CRSwNP groups that we found in this study: Actinobacteria, Bacteroidetes, Cyanobacteria, Firmicutes, Fusobacteria, and Proteobacteria but each group had different percentages of each phylum. In the CRSsNP group, Bacteroidetes $(24.15 \%)$ was the higher percentage phyla, while Actinobacteria (6.99\%) was the lowest percentage phyla. Meanwhile, in the 
CRSwNP group, Proteobacteria (54.86\%) was the higher percentage phyla, while Bacteroidetes (3.37\%) was the lowest percentage phyla.

In our study, Actinobacteria phylum was considered as sinonasal commensal organisms that seem to be having a lower percentage in both groups (CRSsNP; 6.99\%, CRSwNP; 4.05\%). This phylum is identified as "Gatekeepers" 20 to maintain the health condition of the sinonasal environment. Surprisingly, the phyla Proteobacteria had a high percentage in both groups, in the CRSwNP group, this

Table 4: Percentage of the dominant microbiome at phyla level of CRSsNP and CRSwNP groups

\begin{tabular}{llll}
\hline Phylum & CRSsNP & Phylum & CRSWNP \\
\hline Bacteroidetes & $24.15 \%$ & Proteobacteria & $54.86 \%$ \\
Proteobacteria & $22.65 \%$ & Firmicutes & $21.06 \%$ \\
Cyanobacteria & $18.83 \%$ & Cyanobacteria & $12.51 \%$ \\
Firmicutes & $15.50 \%$ & Fusobacteria & $4.15 \%$ \\
Fusobacteria & $11.88 \%$ & Actinobacteria & $4.05 \%$ \\
Actinobacteria & $6.99 \%$ & Bacteroidetes & $3.37 \%$ \\
Total & $100 \%$ & Total & $100 \%$ \\
\hline
\end{tabular}

phylum dominating the microbiome composition (54.86\%), while in the CRSsNP group, this phylum also had a high percentage (22.65\%). The previous study explains the abundance of these phyla correlated with asthmatics subjects. ${ }^{8,18}$ We speculated the result of our current study correlated with these conditions.

Pathogen organism, Fusobacteria seen in both groups, but in a low percentage in CRSwNP (4.15\%). These phyla have high virulence and are frequently involved in severe infections, whether this pathogen had a possible role in the disease process in CRSsNP, compared with CRSWNP, as a cause or only assist the progression of the disease cannot be seen in our study.

In our study, the CRSsNP group had greater bacterial abundance compared with the CRSwNP group, but reduced species richness and diversity.

Disruption of baseline microbiome (dysbiosis) is regarded to have a role in disease mechanism, reduced diversity, and abundance increasing had correlation with chronic inflammation. The dysbiosis hypothesis has been suggested as a disease mechanism in CRS. Breakdown of baseline microbiome which maintains a healthy, stable state of the sinonasal cavity. The myriad factor can contribute to the alteration of the sinonasal microbiome including host and

\begin{tabular}{|c|c|c|c|c|c|}
\hline \multicolumn{6}{|l|}{ Anosim results } \\
\hline \multicolumn{2}{|l|}{ Group $\triangleq$} & \multicolumn{3}{|c|}{ R-value } & P-value \\
\hline \multicolumn{2}{|c|}{ CRSwNO-CRSsNP } & \multicolumn{3}{|c|}{-0.002} & 0.461 \\
\hline $14<4$ & Page & 1 & of 1 & $\mapsto \triangleright 1$ & 10 \\
\hline
\end{tabular}

\begin{tabular}{|c|c|c|c|c|c|c|}
\hline \multicolumn{7}{|l|}{ MRPP test } \\
\hline Group $*$ & \multicolumn{2}{|l|}{ A } & \multicolumn{2}{|c|}{ Observed-delta } & Expected-delta & Significance \\
\hline CRSwNO-CRSsNP & \multicolumn{2}{|l|}{-0.0024} & \multicolumn{2}{|c|}{0.8052} & 0.8033 & 0.597 \\
\hline B & $4<4$ & Page & of 1 & $\Rightarrow>1$ & 10 & View $1-1$ of 1 \\
\hline
\end{tabular}

Figs 7A and B: (A) ANOSIM (B) MRPP analysis microbial communities structure among groups. * Mean the significantly different between groups $(p<0.05)$
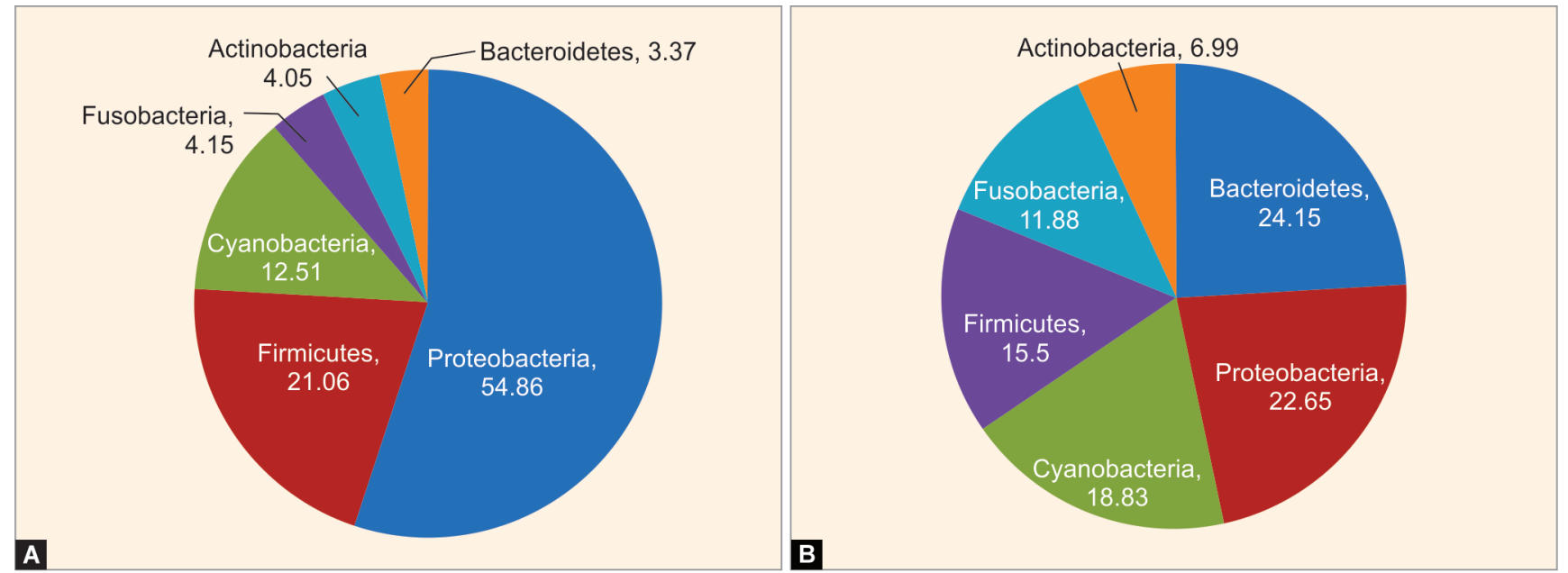

Figs 8A and B: (A) Microbial composition at the phylum level of the CRSsNP group indicates the most abundant phyla detected in the CRSsNP group; (B) Microbial composition at the phylum level of the CRSwNP group indicates the most abundant phyla detected in the CRSwNP group 


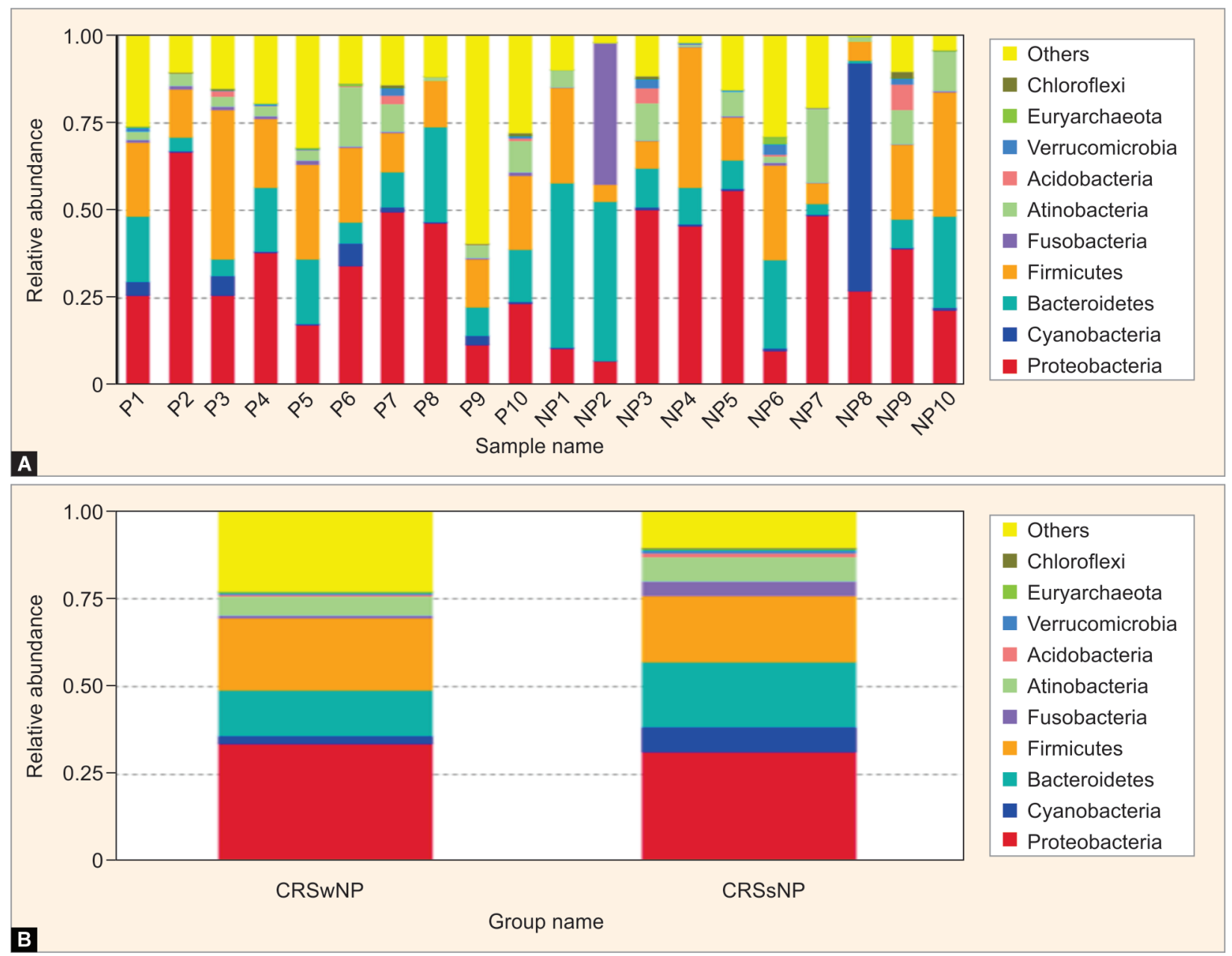

Figs $9 \mathrm{~A}$ and B: Microbial community compositions at the phylum level. Each color represents the percentage of the phylum in the total taxon tags of each sample. The relative abundance of the main bacterial communities found in each sample (A) and groups (B)

environmental. This complex mechanism is still unclear but the role of the microbiome in influencing mucosal health is increasingly important.

Finally, the disparity result of our study from others is likely due to differences in sequence processing, sampling sites, and technique.

Our study showed microbiome profiling with an advanced culture-independent technique (next-generation sequencing). Alteration composition of the microbiome in the sinonasal cavity might have a role in chronic inflammation leading to a disease state. Further investigation involved healthy subjects, larger participants, and a deeper sampling site might reveal more results to obtain a more accurate concept of sinus microbiome in CRS.

In our study, we found that there are five dominant phyla of the microbiome in both groups which are Actinobacteria, Bacteroidetes, Firmicutes Fusobacteria, and Proteobacteria. The study revealed that there is an increase of relative abundance and decrease of diversity in the CRSsNP group indicated dysbiosis phenomenon. No significant differences in alpha diversity of both groups, beta diversity showed a negative $R$ value $(-0.002)$ indicates that inner-group variation is larger than intergroup variations; therefore, no significant differences ( $p$ value $0.461>0.05$ ) between the CRSsNP and CRSwNP groups.

\section{References}

1. Acute and chronic sinusitis. In: Chan Y, Goddard J, ed. K. J. Lee's Essential Otolaryngology Head and Neck Surgery. 11th ed., New York: McGraw Hill; 2016. pp. 491-509.

2. Fokken WJ, Lund VJ, Mullol J, et al. EPOS 2012: European position paper on rhinosinusitis and nasal polyps 2012. A summary for otorhinolaryngologist. Rhinology 2012;50(1):1-12.

3. Bachert C, Pawankar R, Zhang L, et al. ICON: chronic rhinosinusitis. World Allergy Organ J 2014;25(7):25. DOI: 10.1186/1939-4551-7-25.

4. Anderson M, Stokken J, Sanford T, et al. A systematic review of the sinonasal microbiome in chronic rhinosinusitis. Am J Rhinol Allergy 2016;30(3):162-166. DOI: 10.2500/ajra.2016.30.4320.

5. Stevens WW, Lee RJ, Schleimer RP. Chronic rhinosinusitis pathogenesis. J Allergy Clin Immunol 2015;136(6):1442-1453. DOI: 10.1016/j.jaci.2015.10.009.

6. Sivasubramaniam R, Douglas R. The microbiome and chronic rhinosinusitis. World J Otorhinolaryngol Head Neck Surg 2018;4(3):216-221. DOI: 10.1016/j.wjorl.2018.08.004.

7. Green RJ, Niekerk AV, Jeevarathnum AC, et al. The microbiome in chronic inflammatory airway disease: a threatened species. S Afr Med J 2016;106(8):779-781. DOI: 10.7196/SAMJ.2016.v106i8. 11159.

8. Mahdavinia M, Keshavarzian A, Tobin M. A comprehensive review of the nasal microbiome in chronis rhinosinustis (CRS). Clin Exp Allergy 2016;46(1):21-41. DOI: 10.1111/cea.12666. 


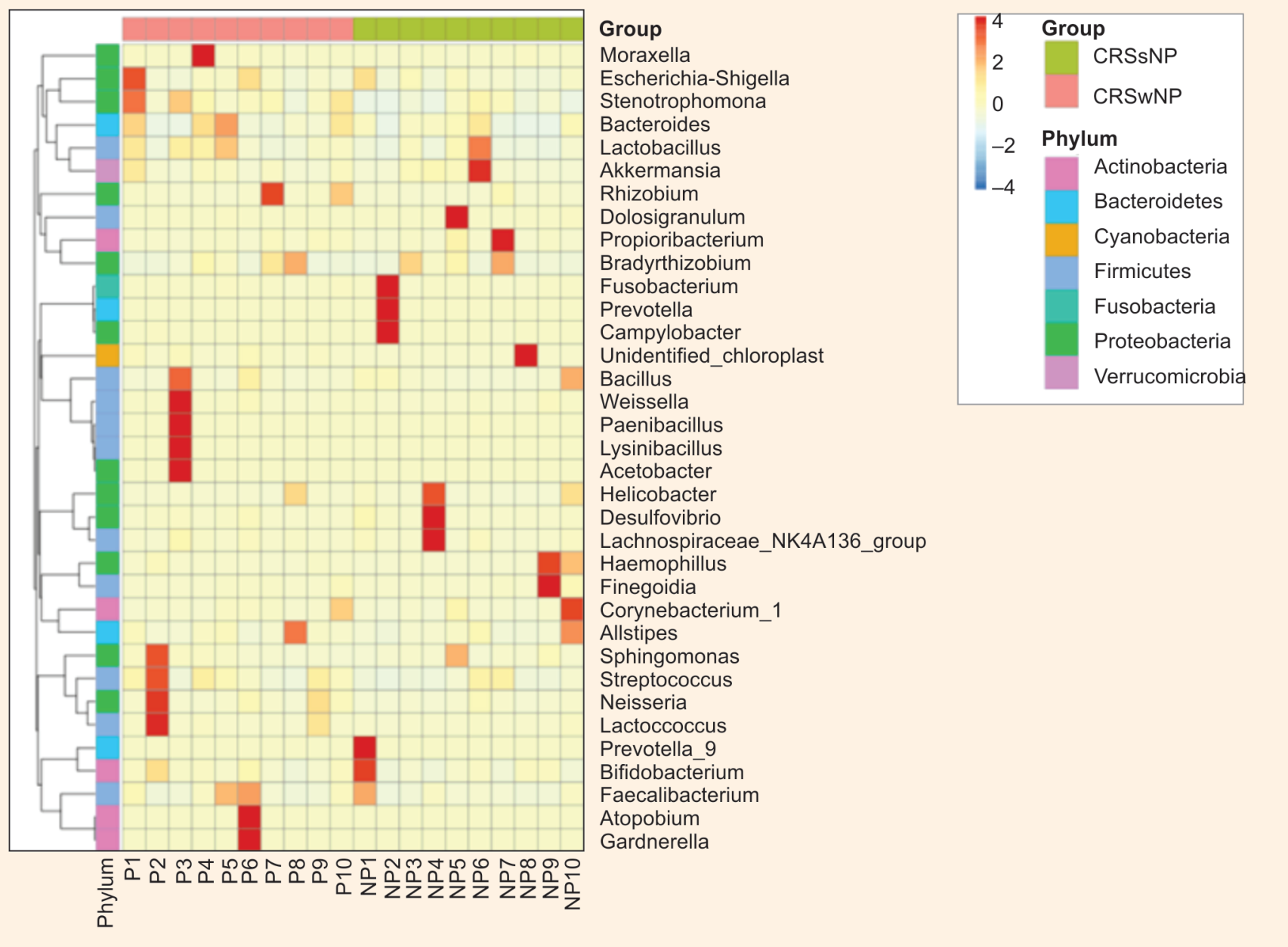

Fig. 10: Clustered heat-map of the microbial communities in two groups. The heat-map delineates the relative percentage of each genus within each sample (horizontal clustering). The relative values for each genus indicated by the color intensity with the legend indicated at the right of the figure

9. Ramakrishnan V, Hauser LJ, Frank DN. The sinonasal bacterial microbiome in health and disease. Curr Opin Otolaryngol Head Neck Surg 2016;24(1):20-25. DOI: 10.1097/MOO.0000000000000221

10. Copeland E, Leonard K, Carney R, et al. Chronic rhinosinusitis: potential role of microbial dysbiosis and recommendations for sampling sites. Front Cell Infect Microbiol 2018;8:57. DOI: 10.3389/ fcimb.2018.00057.

11. Chalerwatanachai T, Velasquez LC, Bachert C. The microbiome of upper airways: focus on chronic rhinosinusitis. World Allergy Organ J 2014;8(3):3.

12. Lozupone CA, Hamady M, Kelley ST, et al. Quantitative and qualitative betadiversity measures lead to different insights into factors that structure microbial communities. Appl Environ Microbiol 2007;73(5):1576-1585. DOI: 10.1128/AEM.01996-06.

13. Koeller K, Herlemann DPR, Schuld T, et al. Microbiome and cultured based analysis of chronic rhinosinusitis compared to healthy sinus mucosa. Front Microbiol 2018;9:643. DOI: 10.3389/fmicb.2018 .00643 .

14. Boase S, Foreman A, Cleland E, et al. The microbiome of chronic rhinosinusitis: culture, molecular diagnostics and biofilm detection. BMC Infect Dis 2013;13:210. DOI: 10.1186/1471-233413-210.
15. Cleland EJ, Bassiouni A, Vreugde S, et al. The bacterial microbiome in chronic rhinosinusitis: richness, diversity, post operative changes, and patient outcomes. Am J Rhinol Allergy 2016;30(1):37-43. DOI: 10.2500/ajra.2016.30.4261.

16. Hoggard $\mathrm{M}$, Biswas K. Evidence of microbiota dysbiosis in chronis rhinosinusitis. Int Forum Allergy Rhinol 2016;7(3):230-239. DOI: 10.1002/alr.21871.

17. Ramakrishnan VR, Feazel LM, Gitomer SA, et al. The microbime of the middle meatus in healthy adults. Plos One Journal 2013;8(12):1-10. DOI: $10.1371 /$ journal.pone.0085507.

18. Ramakrishnan V, Hauser LJ, Feazel LM, et al. Sinus microbiota varies among chronic rhinosinusitis phenotypes and predicts surgical outcome. J Allergy Clin Immunol 2015;136(2):334-42.e1. DOI: 10.1016/j.jaci.2015.02.008.

19. Aurora R, Chatterjee D, Hentzleman J, et al. Contrasting the microbiome from healthy volunteers and patients with chronic rhinosinusitis. JAMA Otolaryngology Head and Neck Surgery 2013;139(12):1328-1338. DOI: 10.1001/jamaoto.2013.5465.

20. Mackenzie BW, Waite DW, Hoggard M, et al. Bacterial community collapse: a meta-analysis of the sinonasal microbiota in chronic rhinosinusitis. Environ Microbiol 2017;19(1):381-392. DOI: 10.1111/14622920.13632. 